\title{
A $75-\mathrm{GHz}$ Phase-Locked Loop in 90-nm CMOS Technology
}

\author{
Jri Lee, Member, IEEE, Mingchung Liu, and Huaide Wang
}

\begin{abstract}
The design and experimental verification of a $75-\mathbf{G H z}$ phase-locked loop (PLL) fabricated in 90-nm CMOS technology is presented. The circuit incorporates a three-quarter wavelength oscillator to achieve high-frequency operation and a novel phase-frequency detector (PFD) based on SSB mixers to suppress the reference feedthrough. The PLL demonstrates an operation range of $320 \mathrm{MHz}$ and reference sidebands of less than $-72 \mathrm{dBc}$ while consuming $88 \mathrm{~mW}$ from a $1.45-\mathrm{V}$ supply.
\end{abstract}

Index Terms-Frequency divider, phase and frequency detector (PFD), phase-locked loop (PLL), reference spurs, transmission line, voltage-controlled oscillator (VCO).

\section{INTRODUCTION}

$\mathbf{T}$ HE TREND for pursuing higher data rate in modern communication systems has been demonstrated in the past and will continue in the future. Recent research on $60-\mathrm{GHz}$ wireless transceivers [1], [2] reveals the advantage of utilizing the 7-GHz unlicensed band and provides possible CMOS realization for the millimeter-wave front ends. Meanwhile, the wireless applications targeting even higher frequencies are also emerging gradually, such as $77-\mathrm{GHz}$ anticollision systems, adaptive cruise control, and $94-\mathrm{GHz}$ Doppler cloud radars. Similar situation can be found in wireline, where broadband data communications are moving toward $20 \mathrm{~Gb} / \mathrm{s}$ (backplane transceivers), $40 \mathrm{~Gb} / \mathrm{s}$ (optical links), and $100 \mathrm{~Gb} / \mathrm{s}$ (next generation's Ethernet). These approaches all need high-speed clocks, making phase-locked loops (PLLs) continue to play critical roles in the world of communication.

As the size scales down, the CMOS devices have achieved a much higher operation frequency, which is sometimes commensurate with the speed of its bipolar counterparts. The low-power, high-integration characteristics as well as recent improvement on broadband techniques make CMOS attractive in ultra-fast PLL designs. To gain more insight into the evolution trend, we summarize the operation frequency of the fully integrated PLLs published in the literature for the past two decades (Fig. 1). It can be clearly shown that the speed of the CMOS circuits raises up by approximately three orders of magnitude, well exceeding the improvement of the device transit frequency $f_{T} \cdot{ }^{1}$ In other words, the overall progress of performance depends not only on the technology, but more importantly, the circuit technique.

Manuscript received March 29, 2007; revised February 14, 2008.

The authors are with the Electrical Engineering Department, National Taiwan University, Taipei, Taiwan 10617, R.O.C. (e-mail: jrilee@cc.ee.ntu.edu.tw).

Digital Object Identifier 10.1109/JSSC.2008.922719

${ }^{1}$ The $f_{T}$ of $90-\mathrm{nm}$ CMOS is around $100 \mathrm{GHz}$, whereas that of a $1-2-\mu \mathrm{m}$ device is on the order of a few GHz.

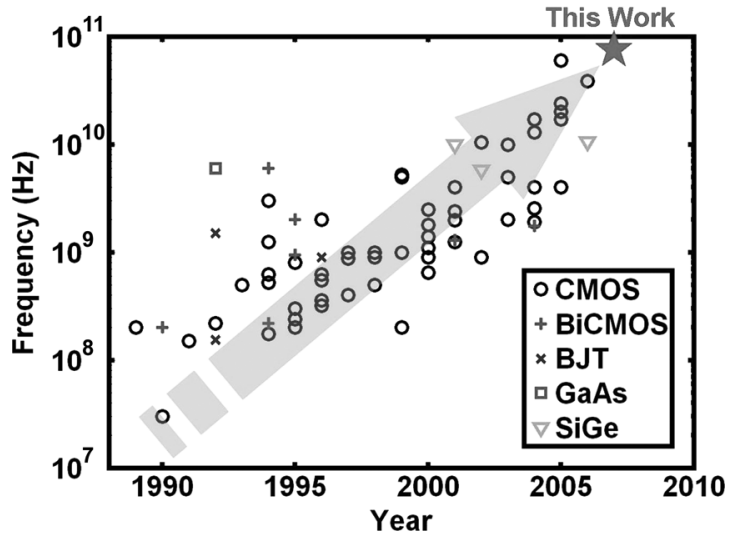

Fig. 1. Evolution of PLL circuit.

It thus inspires us to further explore the limit of the CMOS technology and design a PLL running at even higher frequency.

However, to design such an ultra-high-speed PLL implies a robust VCO together with properly arranged dividers, whose operation locking ranges need to be overlapped with each other. At such a high frequency, connecting blocks with perfect frequency alignment in a loop is much more challenging than making blocks individually. It is because any unexpected parasitic may cause significant frequency shift in the VCO or dividers, prohibiting the loop from lock. Reference spurs, on the other hand, have been another problematic issue in charge pump PLLs. The spurs are primarily due to the pulse-width comparison in the phase detectors, which translates periodic perturbation to the control line. It is well known that this issue leads to serious interferences in the adjacent channels of a wireless system.

In this paper, we present a fully integrated PLL circuit in 90-nm CMOS technology tackling these two issues. Originally designed for the 77-GHz band of automotive radars, this PLL operates at a frequency slightly lower than the desired value. The circuit uses a novel VCO design to alleviate the effective loading and achieve a high resonance frequency, and a specially designed bias circuit rejects the noise and coupling from the supply. Appropriate arrangement for different divider topologies relaxes the stringent tradeoff between operation speed and locking range. Meanwhile, a new phase and frequency detector (PFD) topology based on single-sideband (SSB) mixers is proposed to obviate the generation for pulses, leading to a truly "quiet" phase comparison and substantially suppressing the reference sidebands. To the authors' best knowledge, it is currently the fastest fully integrated PLL for all technologies.

This paper is organized as follows. Section II describes the PLL architecture. Section III presents the design and analysis of 


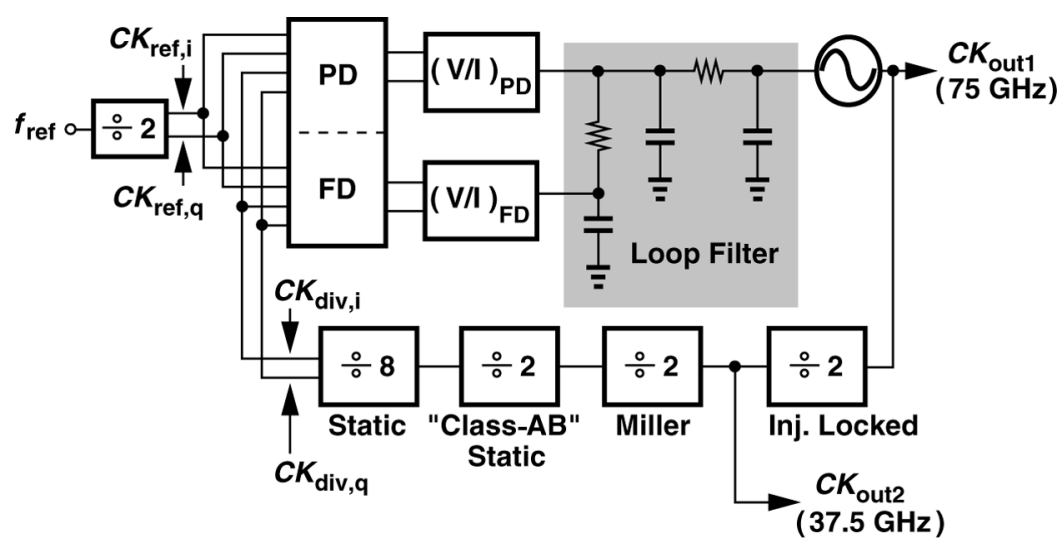

Fig. 2. PLL architecture.

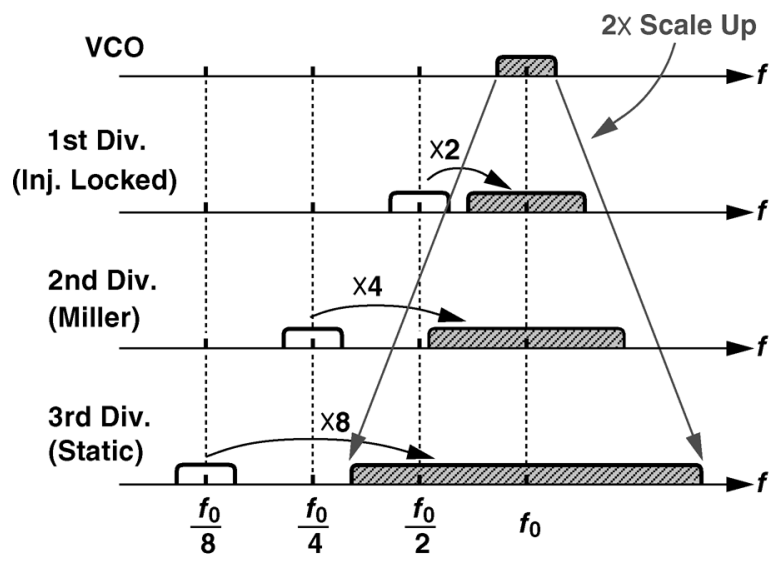

(a)

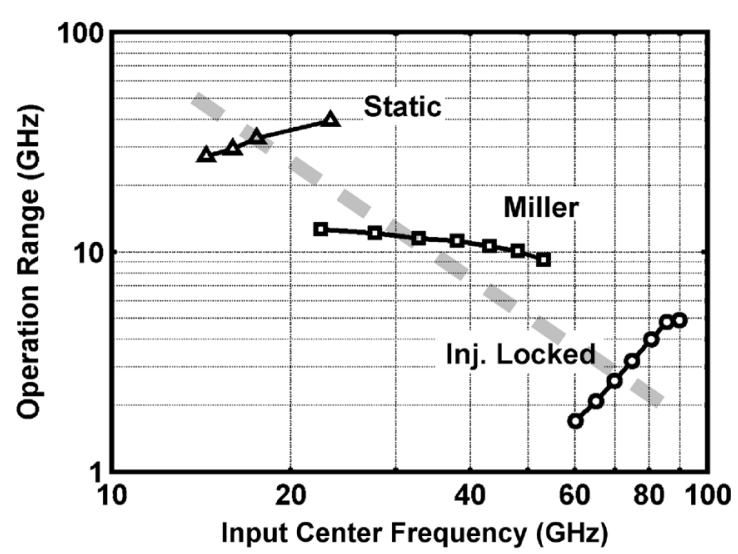

(b)

Fig. 3. Divider arrangement. (a) Locking range normalization with presumed $2 \times$ scale-up requirement. (b) Simulated locking ranges for each divider.

the building blocks, and Section IV summaries the experiment results.

\section{ARCHITECTURE}

Fig. 2 shows the PLL architecture. The PLL circuit consists of a differential VCO, a divider chain with total modulus of 64, a phase and frequency detector, and a third-order loop filter. The phase locking and frequency acquisition loops are decomposed to achieve low jitter and wide operation range simultaneously. The phase and frequency detector is implemented with SSB mixers and low-pass filters to suppress the reference feedthrough. An extra divider-by-2 circuit is incorporated to provide quadrature reference inputs. Similar to that in [3], the frequency detector along with its V/I converter are automatically turned off upon lock to minimize the disturbance to the VCO. The loop filter is realized on chip to minimize the noise coupling through bonding wires. The 9-layer interconnect metals in $90-\mathrm{nm}$ process provide high density fringe capacitor ${ }^{2}$ [4], making the loop filter occupy only $100 \times 300 \mu \mathrm{m}^{2}$.

To accommodate the severe tradeoffs between the input frequency and operation range, different types of dividers need to be employed here. Generally speaking, the injection-locked dividers [5] achieve the highest operation frequency due to the simplest structure, but usually with the narrowest locking

\footnotetext{
${ }^{2} \mathrm{~A}$ structure containing M3-M7 produces a capacitor density of $1.6 \mathrm{fF} / \mu \mathrm{m}^{2}$.
}

range. Static dividers [6], on the other hand, reveal a relatively wide range of operation, but only for low frequencies. Miller dividers [7], also known as regenerative dividers, act as a compromise between the two cases, providing reasonable locking range at moderate center frequency. As a result, a good strategy is to place the injection-locked, Miller, and static dividers in descending order of frequency as the first three stages.

Now we consider the frequency allocation of the VCO and the subsequent dividers. Based on previous design experiences, it is desirable to keep at least $2 \times$ margin for PVT variations to ensure proper locking along the loop. It is because at such high frequencies, even the routing parasitics can lead to significant frequency shift. For example, a $20-\mu \mathrm{m}$ routing path of metal 4 corresponds to 1-2-fF parasitic capacitance, which would cause the center frequency of the first divider stage to drift by $300-500 \mathrm{MHz}$. To ensure proper locking, we would like each divider to have a working range as wide as the VCO tuning range. As depicted in Fig. 3(a), the normalized (i.e., converted to VCO's frequency) locking range scales up by a factor of 2 as the operation frequency decreases. Simulations with different process corners and temperatures verifies this coarse yet useful estimation.

The above requirement is not very difficult to achieve if we select the dividers' topologies correctly. The Miller and static dividers actually exhibit much larger operation ranges in practice, alleviating the design requirement to some extent. Fig. 3(b) plots the simulated operation ranges for three dividers targeting 80 , 

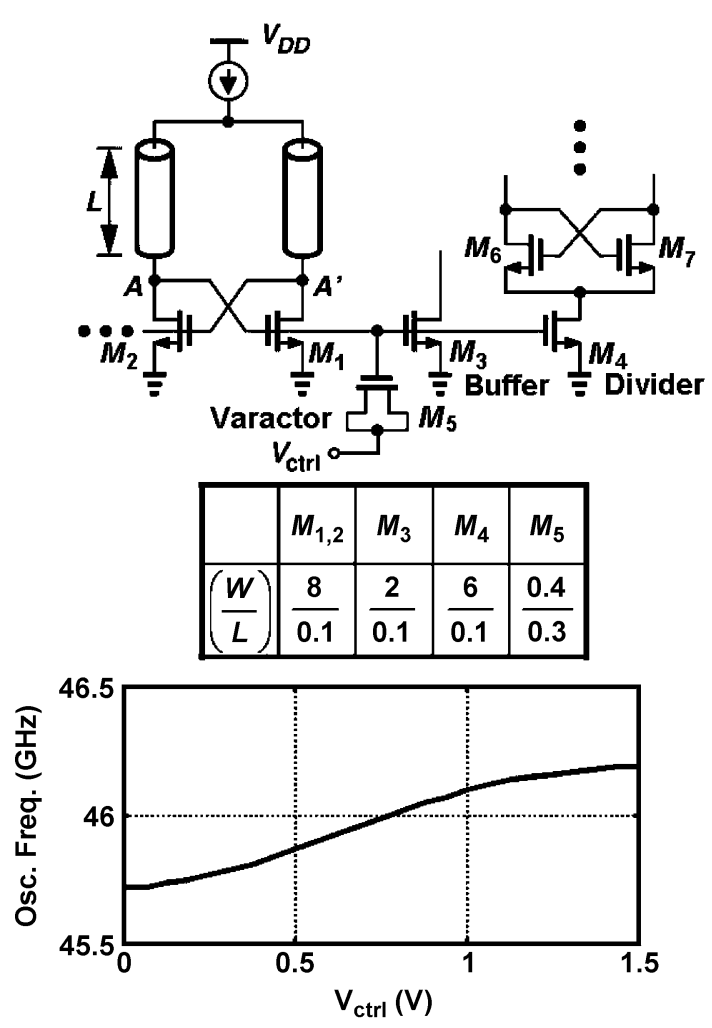

(a)

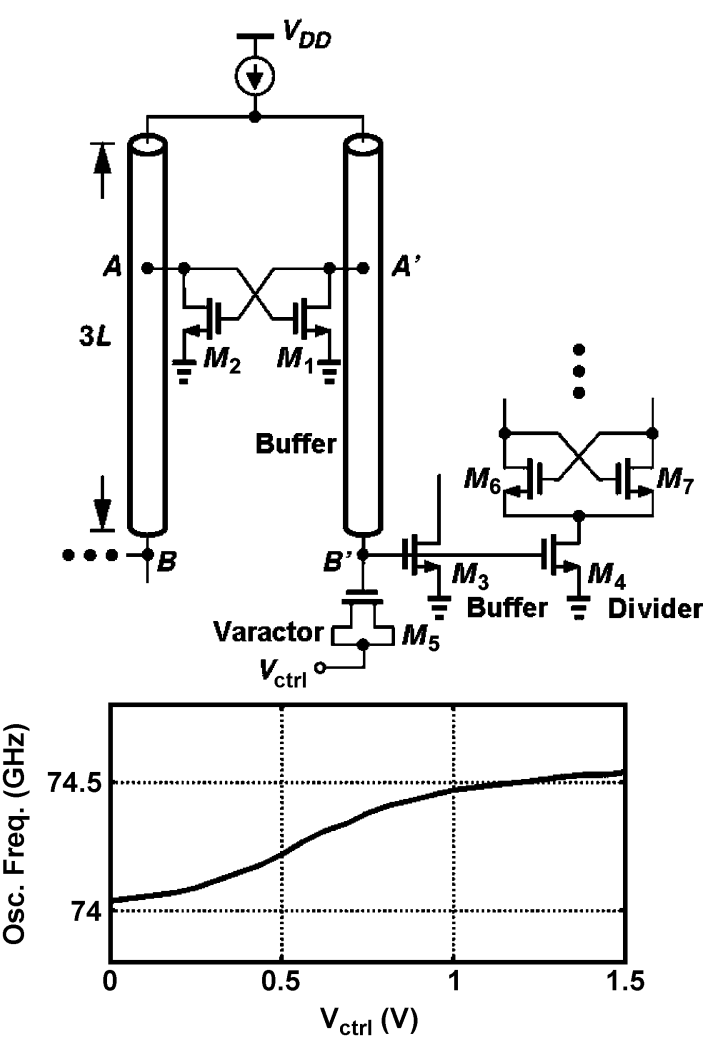

(b)

Fig. 4. (a) Conventional $L C \mathrm{VCO}$, (b) proposed realization with distributed loading.

40, and $20 \mathrm{GHz}$ with injection-locked, Miller and static topologies. ${ }^{3}$ The three curves can be roughly aligned (as the bold dash line), suggesting a direct tradeoff between the input frequency and the operation range since the product of the two is approximately a constant. Note that we design and optimize the dividers for a certain input frequency first, and then exam the corresponding bandwidth. That explains why we can observe a nominally $20-\mathrm{GHz}$ divider with $30-\mathrm{GHz}$ operation range. Nonetheless, subtle loading adjustment and meticulous EM simulations are conducted to ensure strong locking between the VCO and dividers. It is worth noting that the third divider (i.e., the first static stage) is realized as current mode logic (CML) with "class-AB" style to speed up the operation [8]. That is, the tail currents are removed, and the switching is accomplished by gate control. The remaining three dividers at lower speed are implemented as regular CML with proper design to achieve minimum power consumption. We discuss the building blocks in detail in the following section.

\section{BUILDING BLOCKS}

\section{A. $V C O$}

The popular cross-coupled VCO serves as the most suitable candidate for ultra high-speed operation owing to its simplicity. Generally speaking, a resonator can be modeled as a short-circuited quarter-wavelength $(\lambda / 4)$ resonator, regardless

\footnotetext{
${ }^{3}$ The simulation is carried out with a constant input sensitivity of around $4 \mathrm{dBm}$ in $90-\mathrm{nm}$ CMOS.
}

of whether the oscillating "tube" is indeed a transmission line. 4 Fig. 4(a) introduces a typical high-speed VCO design with a simple buffer $\left(M_{3}\right)$, an injection locked divider $\left(M_{4}\right.$ and $M_{6}-M_{7}$ ), and a MOS varactor $\left(M_{5}\right)$. The circuit oscillates at a frequency such that the corresponding wavelength is 4 times as large as the equivalent length $L$, leaving the ends (node $A$ and $A^{\prime}$ ) as maximum swings. However, as the resonance frequency increases, the loading of the varactors, the buffers, and the dividers becomes significant as compared with that of the cross-coupled pair itself. These indispensable capacitances burden the VCO substantially. Note that none of these devices can be made arbitrarily small: $M_{1}-M_{2}$ pair must provide sufficient negative resistance, transistor $M_{4}$ needs to inject large signal current, and $M_{5}$ has to provide enough frequency tuning. With the device dimension listed in Fig. 4(a), the circuit oscillates at only $46 \mathrm{GHz}$. Note that the device sizes have approached the required minimum and further shrinking may cause significant swing degradation.

To overcome the above difficulty, we introduce transmission lines equivalent to three-quarter wavelength $(3 \lambda / 4)$ of a 75-GHz clock to distribute the loading and boost the oscillation frequency. As can be shown in Fig. 4(b), these lines have one end short-circuited and the other open-circuited, resonating differentially with the cross-coupled pair $M_{1}-M_{2}$ providing negative resistance. Connecting to the one-third points of the lines (nodes $A$ and $A^{\prime}$ ), this pair forces the transmission lines to create peak swings at these nodes. The waves thus propagate

${ }^{4}$ Resembling the transmission line, a spiral inductor can always be approximated by a $R L C$ model. 

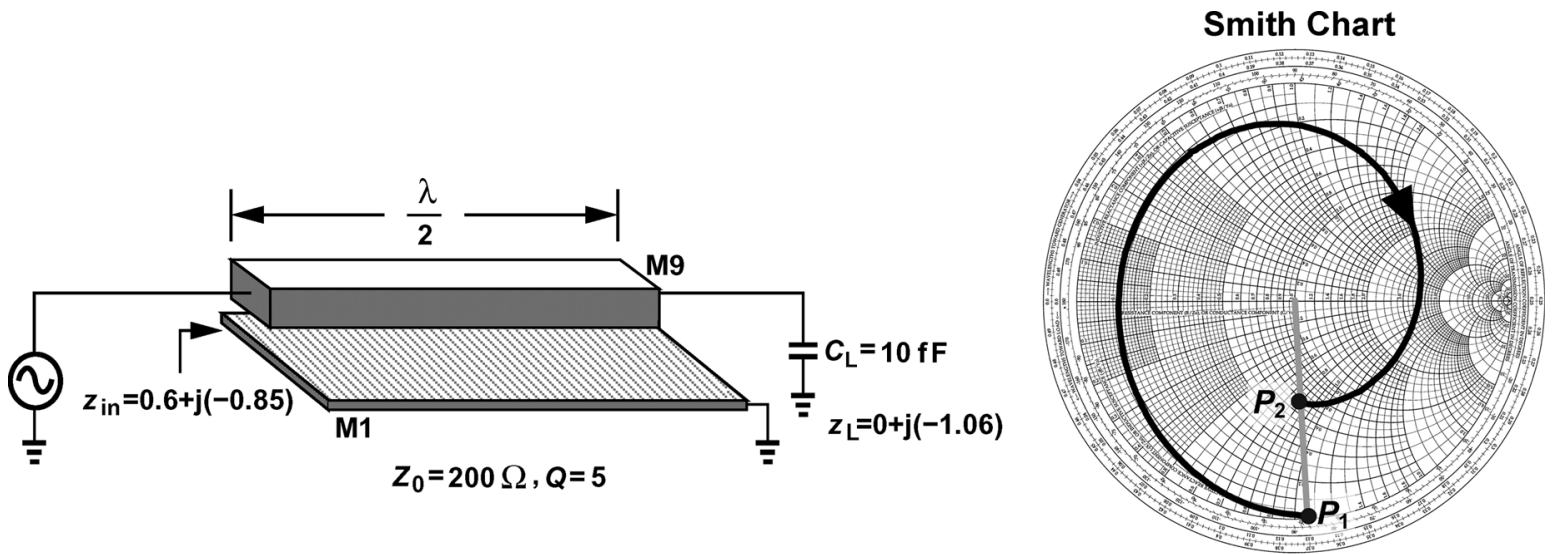

(a)

(b)
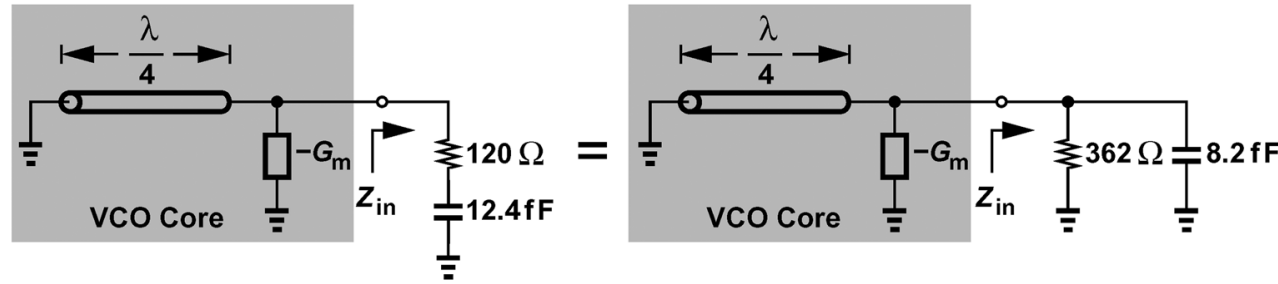

(c)

Fig. 5. Impedance transformation: (a) half-wavelength microstrip line, (b) rotation on Smith chart, (c) series-to-parallel conversion.

and reflect along the lines, forming the second maximum swings with opposite polarities at nodes $B$ and $B^{\prime}$. That is, node $A\left(A^{\prime}\right)$ and node $B\left(B^{\prime}\right)$ are $180^{\circ}$ out of phase. As a result, the buffers, dividers, and varactors can be moved to these ends to relax the loading at nodes $A$ and $A^{\prime}$, making the two zenith positions bear approximately equal capacitance. With the same device dimension $\left[M_{1-5}\right.$ in Fig. 4(a)], the oscillation frequency raises up to around $75 \mathrm{GHz}$, which is a $60 \%$ improvement without any extra power dissipation.

The reader may wonder why the loading capacitance at node $B\left(B^{\prime}\right)$ would look differently at node $A\left(A^{\prime}\right)$. Indeed, the loading at nodes $A\left(A^{\prime}\right)$ and $B\left(B^{\prime}\right)$ will appear identical if the transmission line is lossless, since the $\lambda / 2$ line rotates the loading impedance by exactly $360^{\circ}$ along the outmost circle of the Smith chart. However, in a lossy line the equivalent capacitance seen from node $A\left(A^{\prime}\right)$ toward the load does become lower. The magnitude attenuation translates the purely capacitive loading into a lossy but smaller capacitor. Consider a typical microstrip line with $\lambda / 2$ length as shown in Fig. 5(a). Made of $1-\mu \mathrm{m}$ wide $M_{9}$ on top of $M_{1}$ ground plane, this transmission line would present a characteristic impedance $\left(Z_{0}\right)$ of about $200 \Omega$ and a quality factor $(Q)$ of 5 . Denoting the real and imaginary parts of the propagation constant as $\alpha$ and $\beta$, we have $Q=\beta /(2 \alpha)$ and therefore $\alpha=\pi /(Q \lambda)$. The 10-fF loading capacitor (representing the capacitance of the buffer, the divider, and the varactor) locates at $P_{1}$ with a normalized impedance $z_{L}=0+j(-1.06)$. To calculate the input impedance, we rotate clockwise $z_{L}$ by $360^{\circ}$ with the radius decreasing by a factor of $\exp [-2 \alpha \cdot(\lambda / 2)]$ :

$\exp \left(-2 \alpha \cdot \frac{\lambda}{2}\right)=\exp \left(-2 \cdot \frac{\pi}{Q \lambda} \cdot \frac{\lambda}{2}\right)=\exp \left(-\frac{\pi}{Q}\right)=0.53$

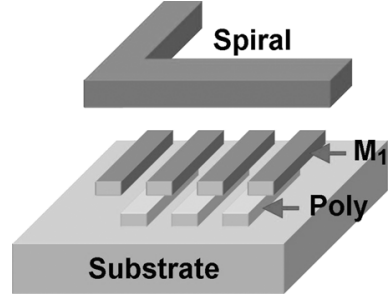

Fig. 6. Arrangement of ground shield.

As depicted in Fig. 5(b), the new location $P_{2}$ represents the normalized impedance $z_{\text {in }}$ which is $0.6+j(-0.85)$. It corresponds to a $12.4 \mathrm{fF}$ capacitor in series with a $120-\Omega$ resistor, which can be further translated to a parallel network $(8.2 \mathrm{fF}$ and $362 \Omega)$ at $75 \mathrm{GHz}$ [Fig. 5(c)]. In other words, the three-quarter wavelength VCO in Fig. 4(b) experiences $18 \%$ less capacitance from $M_{3}-M_{5}$ at a cost of higher loss, which can be compensated by the negative resistance from the cross-coupled pair. Note that the capacitance reduction becomes higher if $Z_{0}$ goes higher.

To achieve high $Q$ and compact layout, the transmission lines are actually realized as three identical inductors in series. As illustrated in Fig. 6, two layers of ground shield made of polysilicon and metall are placed alternately underneath the spirals. Since the gaps are filled, the electric field lines are more confined between the spiral and the shield, minimizing the capacitive coupling to the substrate. Simulations including ASITIC [9] and Sonnet [10] estimate the inductor $Q$ of the VCO to be around 16 at $75 \mathrm{GHz}$.

With the use of spiral inductors, an alternative way to explain the frequency boosting can be found by using the lumped model in Fig. 7. Here, we assume $M_{1}$ and $M_{3-5}$ in Fig. 4 present 


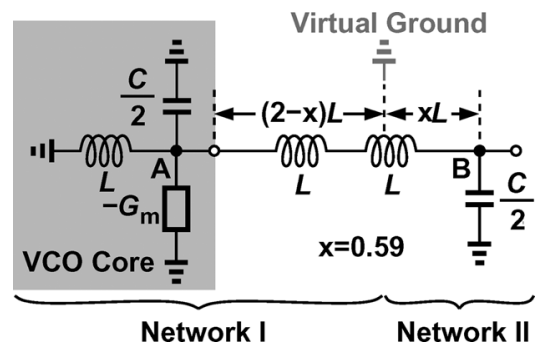

Fig. 7. Frequency estimation of quarter wavelength VCO with lumped model.

equivalent capacitance of $C / 2$ (which is true in our design), and each inductor is denoted as $L$. Since nodes $A$ and $B$ oscillated at the same frequency, there must exist a virtual ground point $x$ located at somewhere along the third inductor such that the Network I and Network II have the same resonance frequency $\omega_{0}$ :

$$
\omega_{0}=\frac{1}{\sqrt{[L \|(2-x) L] \cdot \frac{C}{2}}}=\frac{1}{\sqrt{x L \cdot \frac{C}{2}}} .
$$

It follows that

$$
x=0.59
$$

and

$$
\omega_{0}=\frac{1.84}{\sqrt{L C}} .
$$

Such a first-order model implies a frequency improvement of $84 \%$.

The above analyses also imply that, although the varactors hang on nodes $B$ and $B^{\prime}$, the cross-coupled pair still "sees" the loading variation at these far ends through the two-third segments of the lines. Since the resonance frequency is determined by the inductance of the first one-third segment and the overall equivalent capacitance associated with node $A\left(A^{\prime}\right)$, the tuning of the VCO presents monotonic increasing, similar to that of a regular $L C$ tank VCO. Nevertheless, a standalone VCO with identical structure 5 is developed to verify the observation. As depicted in Fig. 8(a), a monotonically increasing curve of $800 \mathrm{MHz}$ is obtained across $1.2 \mathrm{-V}$ control voltage. In this prototype, we push the VCO frequency to the highest level, which inevitably sacrifices the tuning range to some extent. Fig. 8(b) depicts the VCO tuning over process, temperature, and supply (PVT) variations. The maximum deviation for a given control voltage is about $1.16 \mathrm{GHz}$. For a single chip experiencing $0^{\circ}-85^{\circ}$ temperature change, the oscillation frequency shifts by $0.72 \mathrm{GHz}$. This issue implies that a more advanced technology (e.g., 65-nm CMOS) is necessary for future products operating

\footnotetext{
${ }^{5}$ The tuning of the VCO in the PLL is not measurable here since no pad is connected to the control line. We instead build an individual VCO with identical size but less buffer/routing loading for testing. The oscillation frequency is increased by $10 \mathrm{GHz}$ as expected.
}

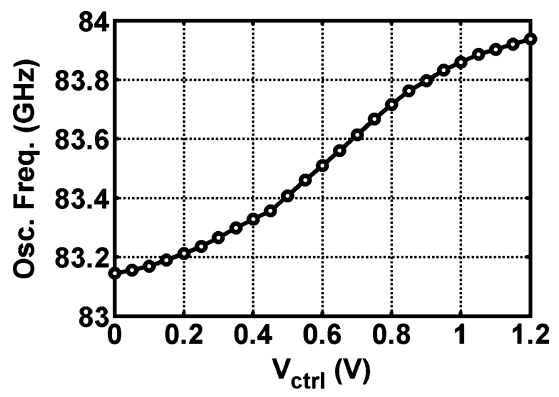

(a)

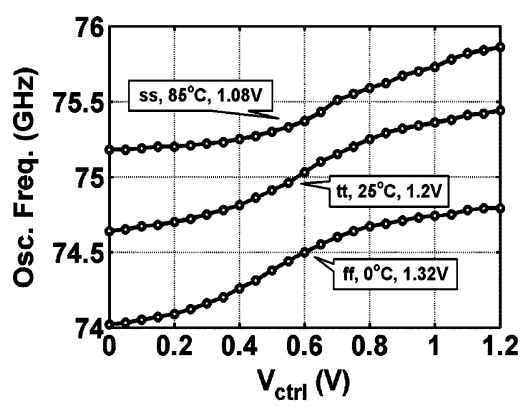

(b)

Fig. 8. (a) Measured tuning curve of a standalone $3 / 4$ wavelength VCO (oscillation frequency is set to be $10-\mathrm{GHz}$ higher than that of the VCO in the PLL), (b) simulated tuning curves for PVT variations.

at this frequency. Fig. 9 shows the simulated waveforms on different nodes of the VCO.

To suppress the coupling from power lines, the VCO is biased with a supply-independent circuit $\left(M_{9}-M_{12}\right.$ and $\left.R_{S}\right)$, as illustrated in Fig. 10(a). Here, we introduce $M_{13}$ to absorb extra current variation caused by channel-length modulation to further reject the supply noise. That is, by proper sizing we set

$$
\left|\frac{\partial I_{\mathrm{SS}}}{\partial V_{\mathrm{DD}}}\right|=\left|\frac{\partial I_{C}}{\partial V_{\mathrm{DD}}}\right|
$$

letting the current flowing into $M_{1}-M_{2}$ pair remain constant [11]. Fig. 10(b) shows the currents through $M_{13}\left(I_{C}\right)$ and $M_{14}\left(I_{\mathrm{SS}}\right)$ as functions of supply voltage, suggesting an equal slope in the vicinity of $1.45 \mathrm{~V}$. In other words, the voltage at node $P$ is fixed, leaving the resonance frequency insensitive to supply perturbation [Fig. 10(c)]. The power penalty of $M_{13}$ can be restrained to as low as $20-30 \%$ with proper design. The performance of this open loop compensation would slightly degrade if PVT variations occur. For example, the supply sensitivity becomes $33.3 \mathrm{MHz} / \mathrm{V}$ and $-53.3 \mathrm{MHz} / \mathrm{V}$ at $1.35-\mathrm{V}$ and $1.55-\mathrm{V}$ supplies, respectively [Fig. 10(c)]. Nonetheless, these results are still much better than that of a conventional design without $M_{13}$. Note that to further reduce the noise from the current source, techniques such as [12] can be applied to this VCO.

\section{B. Frequency Dividers}

As described in Section II, the dividers are implemented as different topologies depending on the operation frequency. The first stage is realized as a simple injection-locked structure, 


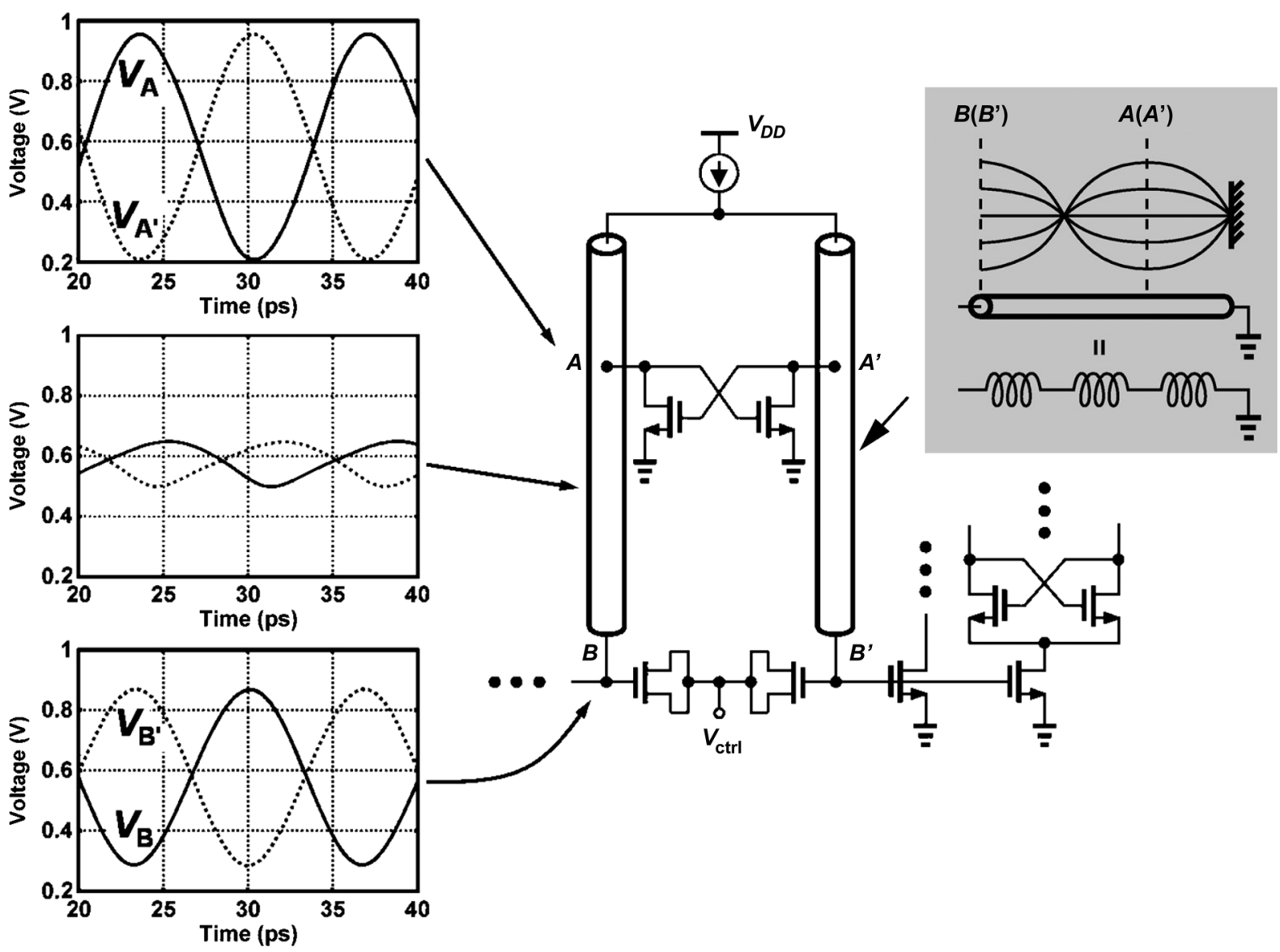

Fig. 9. Simulated VCO waveforms.

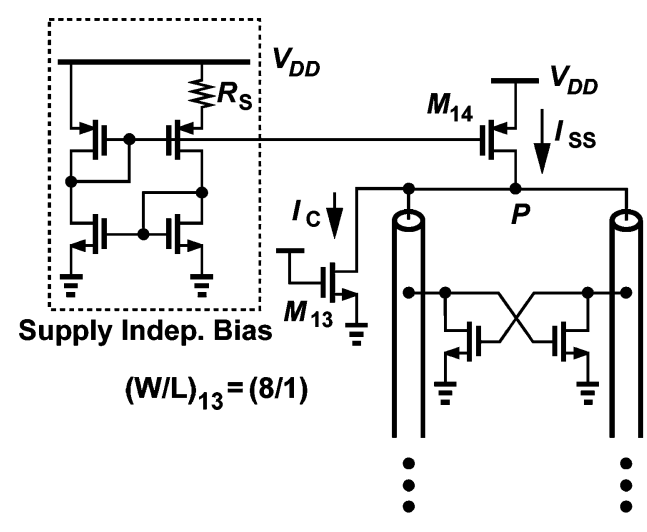

(a)

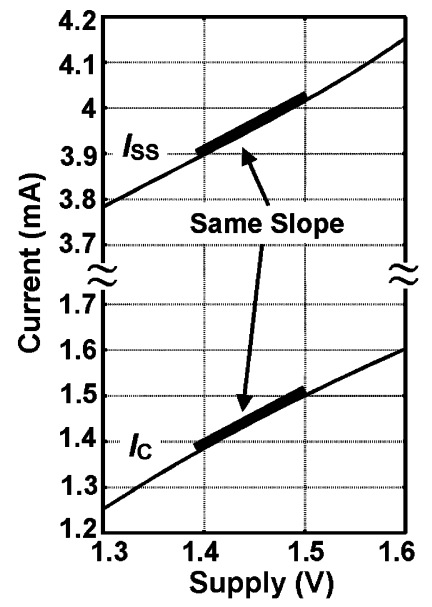

(b)

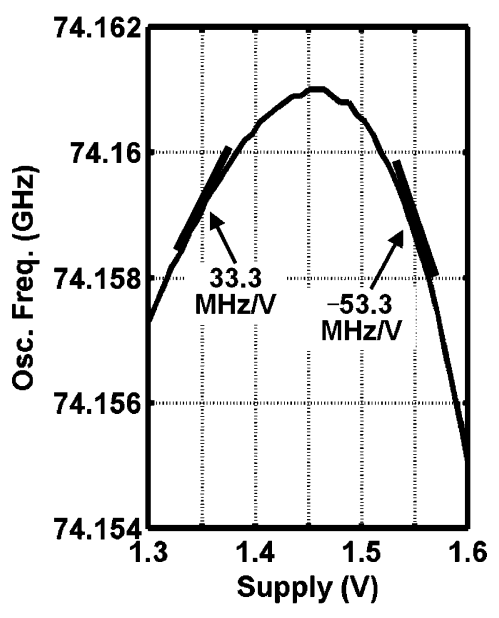

(c)

Fig. 10. (a) Supply independent biasing, (b) current variations, (c) oscillation frequency as a function of supply voltage.

where the natural biasing established by the cross-coupled pair of the VCO facilitates a dc coupling between the two. The complete design of the $\mathrm{VCO}$, the first divider, and the buffer are shown in Fig. 11. Here, two identical injection-locked dividers are used to preserve symmetry. It produces two half-rate outputs: one goes to the second divider stage and the other to an output pad for testing. Dummy buffer is used, that along with careful layout arrives at perfect balance between the loading at nodes $B$ and $B^{\prime}$. Inductor $L_{R}$ is added to resonate out the parasitic capacitance associated with nodes $C$ and $C^{\prime}$, allowing stronger signal injection through $M_{5}$ and $M_{6}$ [7], [13].

The second divider stage is realized as a Miller divider with bandpass load, as depicted in Fig. 12(a). Similar to the second stage in [7], this structure has the output fed back to the LO port and resembles an injection-locked divider with differential input. Simulation reveals a locking range of about $11 \mathrm{GHz}$. With 


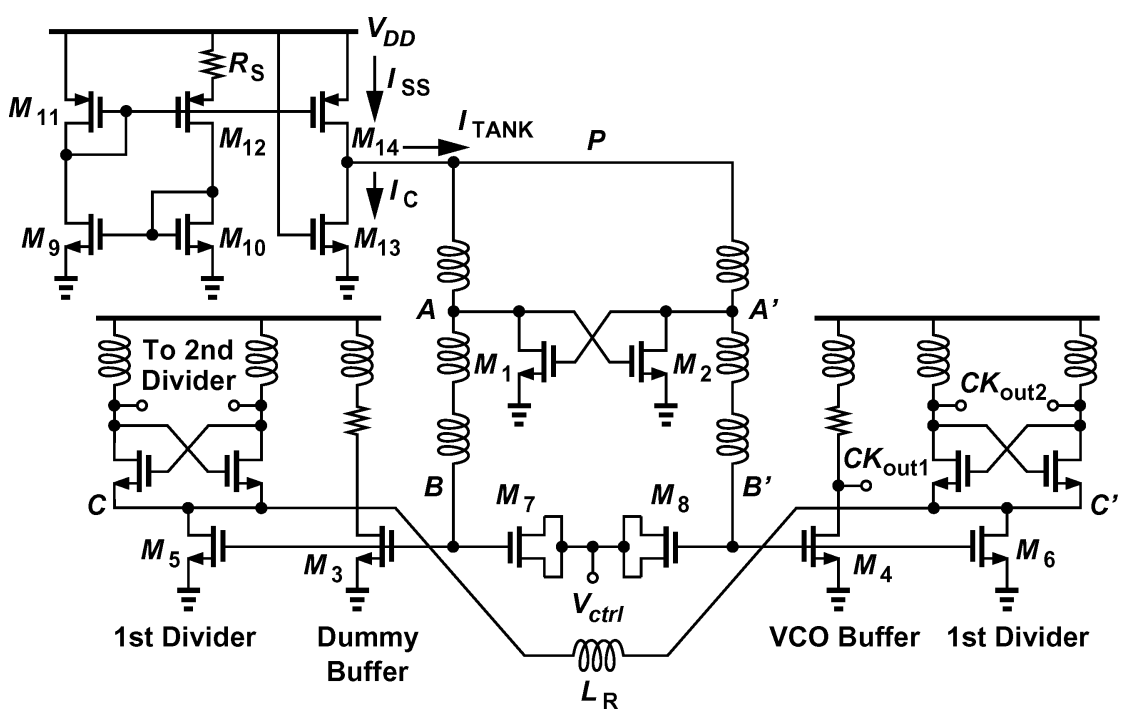

Fig. 11. VCO and first divider.

the first two stages the frequency is brought down to below $20 \mathrm{GHz}$, allowing static implementation on the subsequent dividers. To extend the bandwidth without using inductors, we realize the third divider stage as class-AB structure [Fig. 12(b)]. The large instantaneous currents create high gain in the signal path and therefore higher speed. Beyond this point, the divider design becomes much more relaxed. The succeeding stages are implemented as standard static dividers with the power and bandwidth optimized.

Duo to the limited silicon area available, we are not capable of developing a full library characterizing the high-frequency models for the active and passive devices. We instead design the VCO and the first divider with the following approach. First, a preliminary version of the $\mathrm{VCO}+$ divider codesign based on the foundry models are taped out to estimate the accuracy of the inductors. Second, we gradually reduce the bias current of the test key until the divider fails. It allows us to obtain a coarse design margin. Since the device model below $40 \mathrm{GHz}$ are well established through our previous experience on this technology, the frequency alignment of the whole divider chain could possibly be achieved in the second run. The layout skill is of great importance as well. The differential signal lines are carefully laid out with perfect symmetry so that the mismatch between the full-rate lines is less than $1 \mathrm{fF}$.

\section{Phase and Frequency Detector}

Ever since charge-pump topology became a mainstream in modern PLL designs, the reference clock feedthrough has been a bothering issue, especially in multi-channel applications such as frequency synthesizers. Many attempts have been made to minimize the reference spurs. For example, charge transfer technique spreads out the momentary (positive or negative) increment over longer period [14], [15]; analog phase detector utilizes current-mode logic to reduce swing [3], [16]; compensated charge-pump design balances the device mismatch [17], [18]; and distributed phase detector shortens the step of variation to avoid abrupt changes on the control voltage [19], [20]. However, none of these approaches can really get rid of the pulse generation, so the control line ripple can never be removed entirely.
To avoid producing on-off pulses which potentially create reference spurs, we perform phase detection by mixing two quadrature signals, one from the reference input $\left(C K_{\text {ref }}\right.$, provided by the static divide-by-2 circuit) and the other from the last divider stage $\left(C K_{\text {div }}\right)$. As illustrated in Fig. 13, an SSB mixer can distill the phase error of two synchronous signals, and reveals a sinusoidal input-output characteristic. Driven by the phase detector output $V_{\mathrm{PD}}$, the $\mathrm{V} / \mathrm{I}$ converter provides a continuous and proportional current, either positive or negative, to the loop filter and changes the control voltage accordingly. Since the characteristic can be approximately considered linear in the vicinity of origin and no pulse generation is involved, it achieves a truly "quiet" phase examination and reference spurs are significantly reduced. It is important to know that the current imbalance in the $\mathrm{V} / \mathrm{I}$ converter is no longer an issue here, since the phase detector would create an offset between the two inputs to compensate it perfectly.

In the presence of mismatches, finite "image" would be observed at twice the PD operation frequency $2 \omega_{\text {in }}$. To suppress it, a low-pass filter must be placed right after the SSB mixer. A clever realization is to load the mixer with $R C$ networks $(R=$ $600 \Omega, C=32 \mathrm{pF}$ ) [Fig. 13(c)], which generates a corner frequency of $8.3 \mathrm{MHz}$ and reject the image by more than $40 \mathrm{~dB}$. Note that these low-pass filters have little impact on the overall loop bandwidth, which is designed to be around 2-3 MHz only. Fig. 14 compares the control line fluctuation of several PLLs with the same operation frequency and power dissipation but different PD topologies. The proposed structure reveals a minimum ripple of only $15 \mu \mathrm{V}$, as depicted in the inset, whereas type IV and [3] induce perturbation of 13 and $4 \mathrm{mV}$, respectively.

The periodic characteristic of the phase detector implies a limited capture range. Fortunately, the frequency detection can be accomplished by introducing another SSB mixer, arriving at a wide operation range. As shown in Fig. 15, the two outputs $V_{\mathrm{PD}}$ and $V_{2}$ appear orthogonally in the presence of frequency error:

$$
\begin{aligned}
& V_{1}=V_{\mathrm{PD}}=k A_{1} A_{2} \sin \left(\Delta \omega_{\mathrm{in}} t+\theta\right) \\
& V_{2}=k A_{1} A_{2} \cos \left(\Delta \omega_{\text {in }} t+\theta\right) .
\end{aligned}
$$




\begin{tabular}{|c|c|c|c|c|c|c|}
\hline & $M_{1,2}$ & $M_{3,4}$ & $M_{5-6}$ & $M_{7}$ & $I_{\mathrm{b}}$ & $C_{1}$ \\
\hline$\left(\frac{W}{L}\right)$ & $\frac{8}{0.1}$ & $\frac{16}{0.1}$ & $\frac{8}{0.1}$ & $\frac{8}{0.1}$ & $1.6 \mathrm{~mA}$ & $200 \mathrm{fF}$ \\
\hline
\end{tabular}

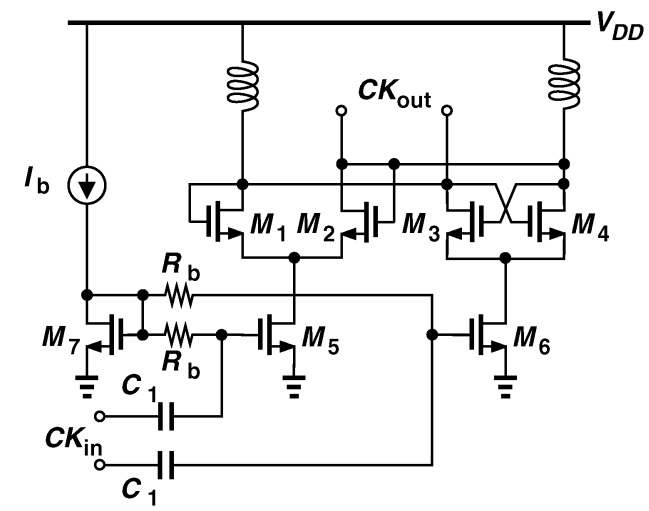

(a)

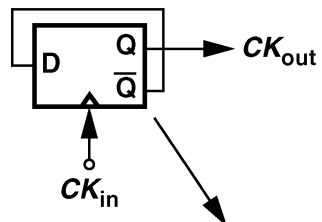

\begin{tabular}{|c|c|c|c|c|c|c|c|}
\hline & $M_{1-4}$ & $M_{5-8}$ & $M_{9-12}$ & $M_{13}$ & $R$ & $I_{\mathrm{b}}$ & $C_{1}$ \\
\hline$\left(\frac{W}{L}\right)$ & $\frac{5}{0.1}$ & $\frac{10}{0.1}$ & $\frac{8}{0.1}$ & $\frac{8}{0.1}$ & $400 \Omega$ & $1 \mathrm{~mA}$ & $250 \mathrm{fF}$ \\
\hline
\end{tabular}

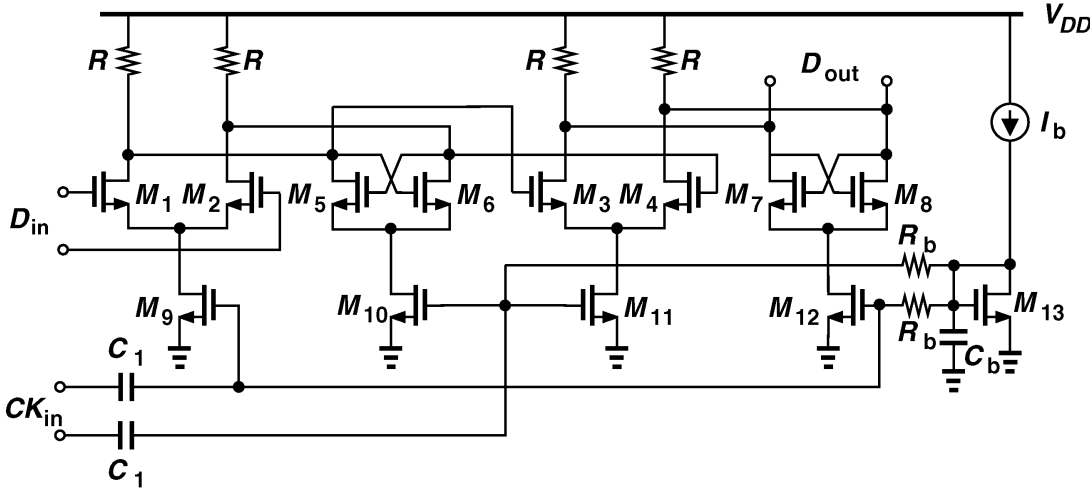

(b)

Fig. 12. (a) Second and (b) third divider stages.

Here, $\Delta \omega_{\text {in }}$ represents the frequency difference between $C K_{\text {ref }}$ and $C K_{\text {div }}$. Obviously, whether $V_{1}$ is leading or lagging $V_{2}$ depends on the sign of $\Delta \omega_{\text {in }}$, and it can be easily obtained by using a flip-flop to sample one signal with the other [3]. Based on the flip-flop's output, the V/I converter designated to the frequency detection loop [i.e., $(\mathrm{V} / \mathrm{I})_{\mathrm{FD}}$ ] injects a positive or negative current to the loop filter. This current is 3 times larger than the peak current of $(\mathrm{V} / \mathrm{I})_{\mathrm{PD}}$ to ensure a smooth frequency acquisition. To minimize the disturbance, the automatic switching-off characteristic [3] is preserved in this design by applying $\overline{E N F D}$ to $(\mathrm{V} / \mathrm{I})_{\mathrm{FD}}$ and making it disabled when the loop is locked. Similar to type IV PFD, this frequency detector can theoretically operate across an infinite range. The relatively simple structure helps to reduce the power consumption.

The very slow sinusoids $V_{\mathrm{PD}}$ and $V_{2}$ may cause malfunction of $\mathrm{FF}_{1}$ if they drive the flip-flop directly, because the transitions of $V_{\mathrm{PD}}$ and $V_{2}$ become extremely slow when the loop is close to lock. The fluctuation caused by unwanted coupling or additive noise would make the transitions ambiguous, possibly resulting in multiple zero crossings. To remedy this issue, hysteresis buffers are employed to sharpen the waveforms. Fig. 16 depicts the buffer design, where the cross-coupled pair $M_{3}-$ $M_{4}$ provides different switching thresholds for low-to-high and high-to-low transitions, and the positive feedback helps to create square waves as well. Here, $(W / L)_{1,2}=(W / L)_{3,4}=8 / 0.25$, and a threshold difference of $46 \mathrm{mV}$ is observed. Fig. 17 shows the complete PFD design.

\section{EXPERIMENTAL RESULTS}

The PLL circuit has been fabricated in 90-nm CMOS technology and tested on a high-speed probe station with dc pads directly wire-bonded to board traces. Fig. 18(a) shows a photo of the die, which measures $1.0 \times 0.8 \mathrm{~mm}^{2}$ including pads, and Fig. 18(b) illustrates the testing setup. In this prototype the reference input is provided by N4901A without any additional filters. 


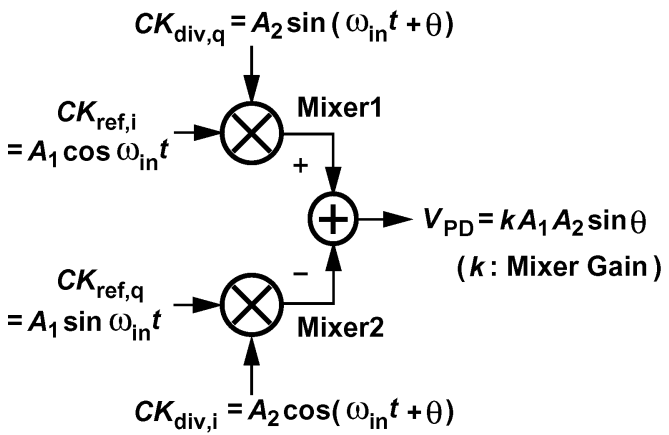

(a)

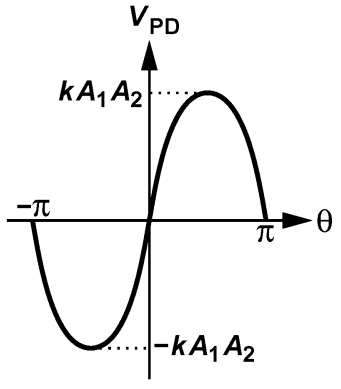

(b)

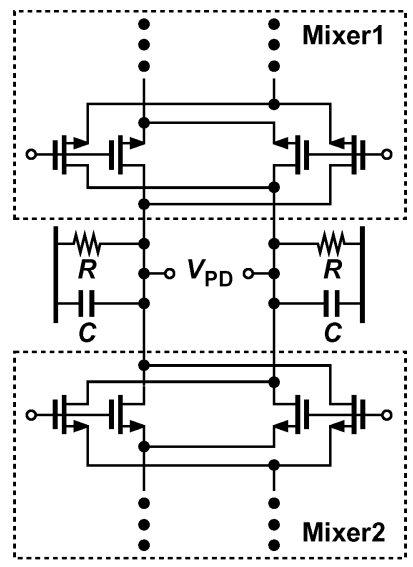

(c)

Fig. 13. (a) Proposed phase detector and (b) its characteristic; (c) SSB mixer with $R C$ low-pass filter.

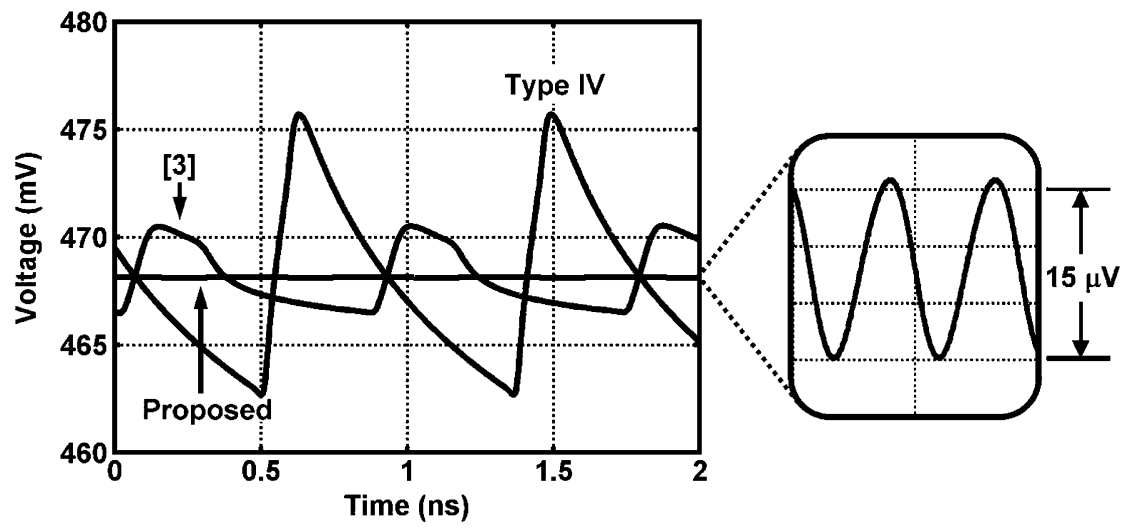

Fig. 14. Simulated control line voltages.

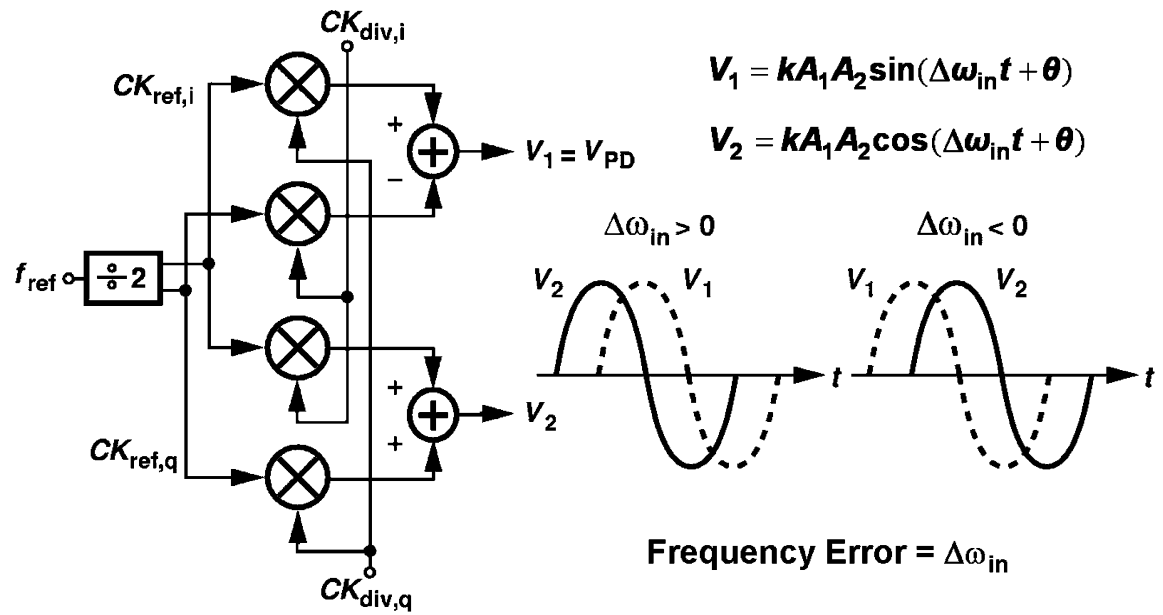

Fig. 15. Frequency detection.

The loop locks from $73.4 \mathrm{GHz}$ to $73.72 \mathrm{GHz}$. The $320 \mathrm{-MHz}$ operation range is pretty much the VCO tuning range. 6 Three dies are tested independently, and the frequency variations are within $50 \mathrm{MHz}$. The PLL functions properly with any supply voltage between $1.35 \mathrm{~V}$ and $1.55 \mathrm{~V}$. The nominal power consumption

${ }^{6}$ Due to the limited silicon area, we do not build standalone dividers for testing. (supply $=1.45 \mathrm{~V}$ ) excluding output buffers is $88 \mathrm{~mW}$, of which $8 \mathrm{~mW}$ is dissipated in the $\mathrm{VCO}, 66 \mathrm{~mW}$ in the divider chain, and $14 \mathrm{~mW}$ in the PFD and V/I converters. The reference input level can be as low as $-16 \mathrm{dBm}$.

At $75 \mathrm{GHz}$, the spectrum analyzer must incorporate harmonic mixer which introduces loss of around $50 \mathrm{~dB}$, resulting in a limited range on phase noise and sideband measurement. Fig. 19(a) 

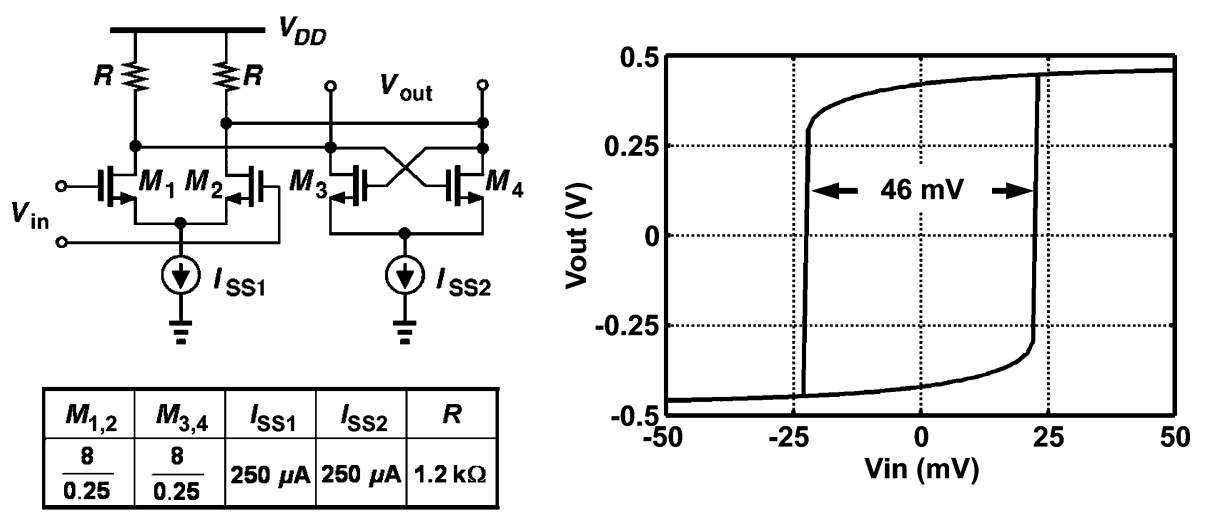

Fig. 16. Hysteresis buffer and its characteristic.

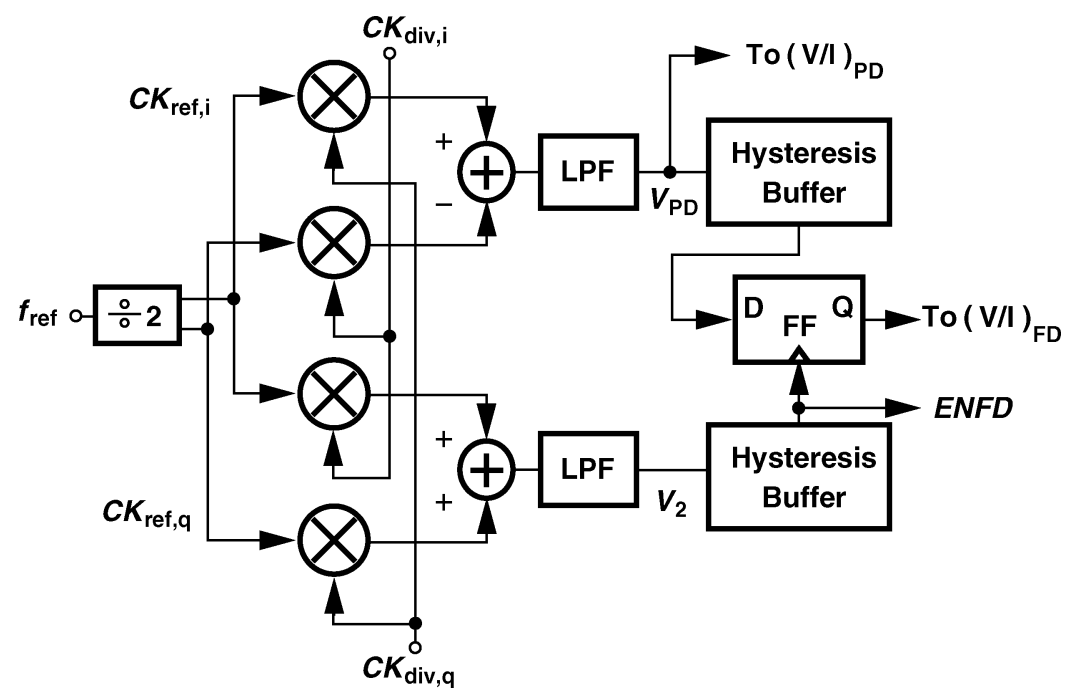

Fig. 17. Complete PFD design.

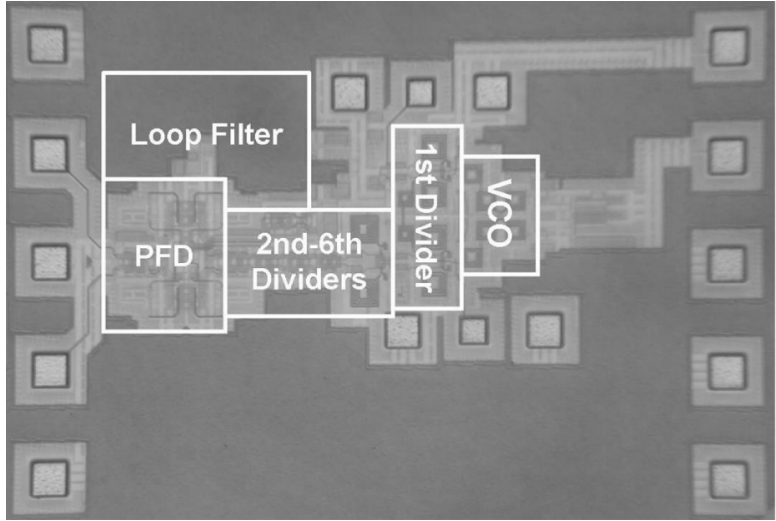

(a)

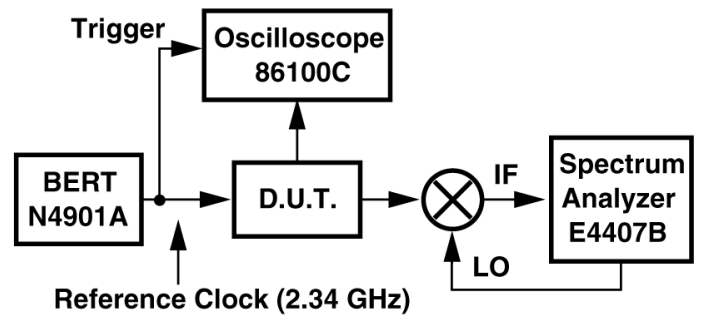

(b)

Fig. 18. (a) Chip micrograph. (b) Testing setup.

depicts the in-band output spectra of the VCO and the first divider under locked condition. The phase noise at $100-\mathrm{kHz}$ offset measures -88 and $-94 \mathrm{dBc} / \mathrm{Hz}$, respectively. The out-of-band spectra are also shown in Fig. 19(b). Here, the heavy loss of the harmonic mixer "raise" the noise floor and make VCO phase noise invisible. The half-rate output presents a phase noise of $-114 \mathrm{dBc} / \mathrm{Hz}$ at $10-\mathrm{MHz}$ offset, suggesting that the $\mathrm{VCO}$ itself has $-108 \mathrm{dBc} / \mathrm{Hz}$ phase noise at $10-\mathrm{MHz}$ offset. The sideband measurement suffers from the same difficulty. We then estimate the reference sideband as follows. First, with the highest resolution, we plot the spectrum of the output and spurs for different input amplitudes as shown in Fig. 20. By gradually turning down the input level, it can be observed that the spurs would eventually buried in the noise floor, which is $72 \mathrm{~dB}$ lower than the carrier, 


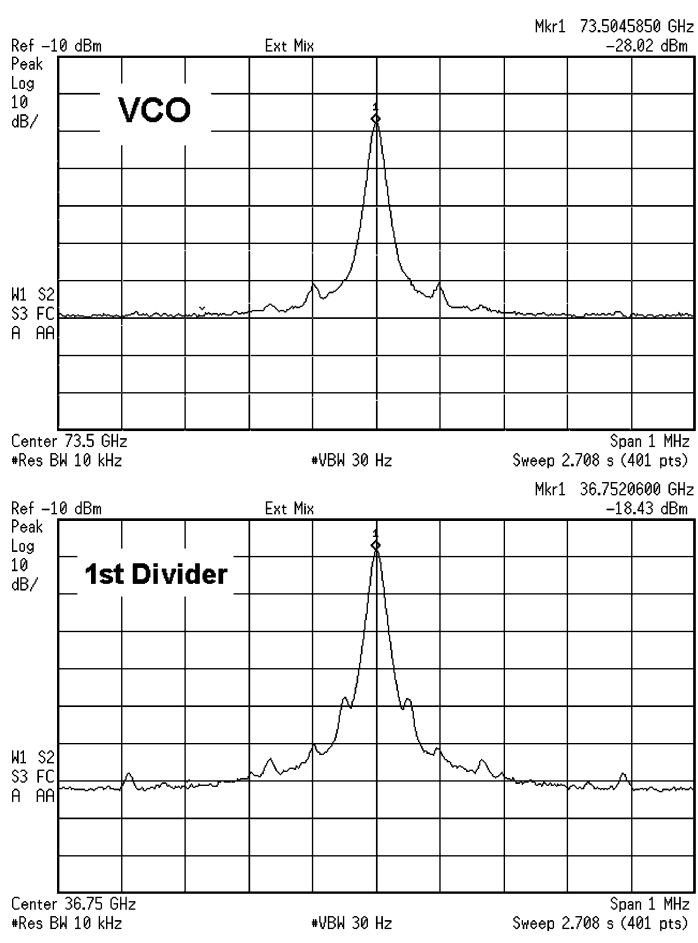

(a)

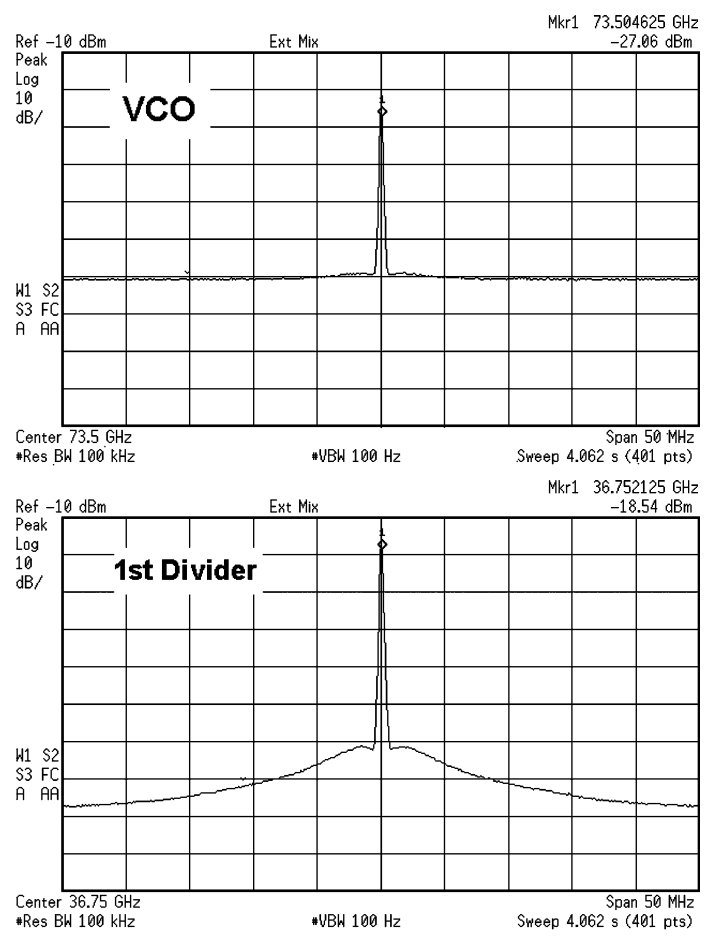

(b)

Fig. 19. (a) In-band, (b) out-of-band output spectra of VCO and the first divider.

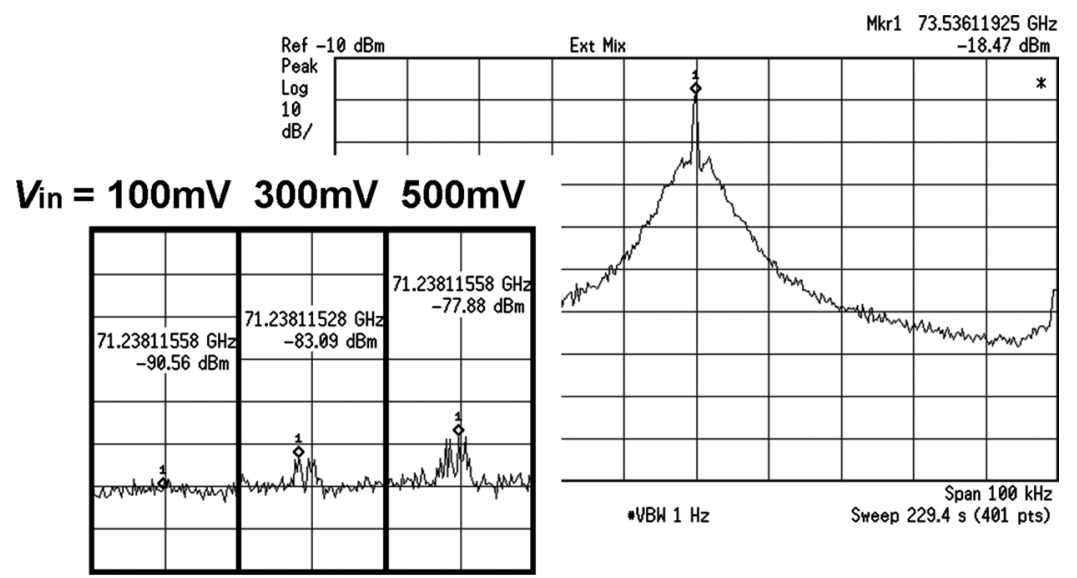

Fig. 20. Measurement on reference spurs.

if the reference input is less than $100 \mathrm{mV}$. Since the PLL can operate perfectly with reference input level as low as $50 \mathrm{mV}$, we conclude that the reference sidebands are less than $-72 \mathrm{dBc}$.

The heavy loss of the mixers (11970W) also prohibits a direct phase noise plot of the $\mathrm{VCO}$, since the spectrum analyzer E4407B requires an input of at least $-50 \mathrm{dBm}$ to do so. We instead depict the phase noise of the first divider's output, and compare it with the input profile as illustrated in Fig. 21. It can be shown that the two curves roughly maintain a difference of $24 \mathrm{~dB}(=20 \log 16)$, suggesting that the PLL itself contributes negligible noise.

The time domain performance are also recorded as shown in Fig. 22. Using the reference clock as a trigger, the $75-\mathrm{GHz}$ output presents peak-to-peak and rms jitter of 609 fs,pp and $87 \mathrm{fs}, \mathrm{rms}$, respectively, and the $37.5-\mathrm{GHz}$ output reveals jitter of $2.15 \mathrm{ps}$,pp and $293 \mathrm{fs}$,rms. The difference might be attributed to the accuracy of the instruments (e.g., the harmonic mixers, sampling modules of the oscilloscope, etc.) at high speed. Nonetheless, integrating the phase noise of the $37.5-\mathrm{GHz}$ output in Fig. 21 from $10 \mathrm{kHz}$ to $10 \mathrm{MHz}$ yields an rms jitter of $372 \mathrm{fs}$, roughly matching the measured result [21]. The loop bandwidth measures $2.5 \mathrm{MHz}$. Fig. 23 compares the reference sidebands of integer- $N$ PLLs with similar loop bandwidth, and Table I summarizes the performance of this work and some other high-speed PLL circuits recently published in the literature. 


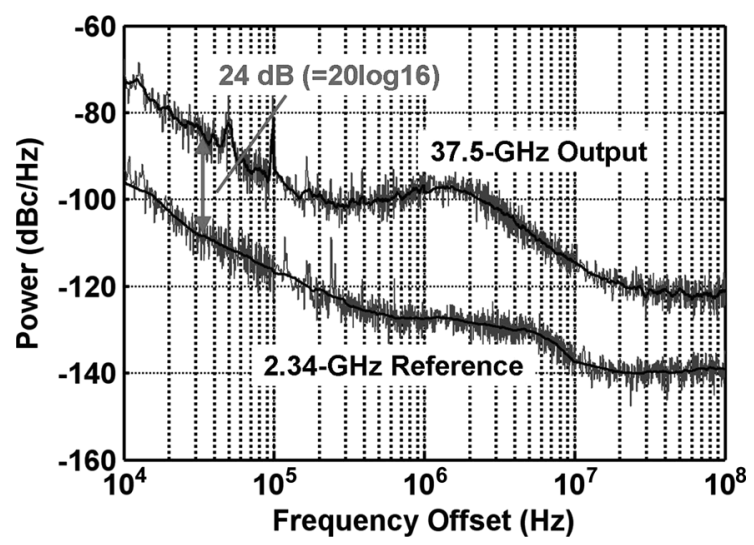

Fig. 21. Phase noise measurement.

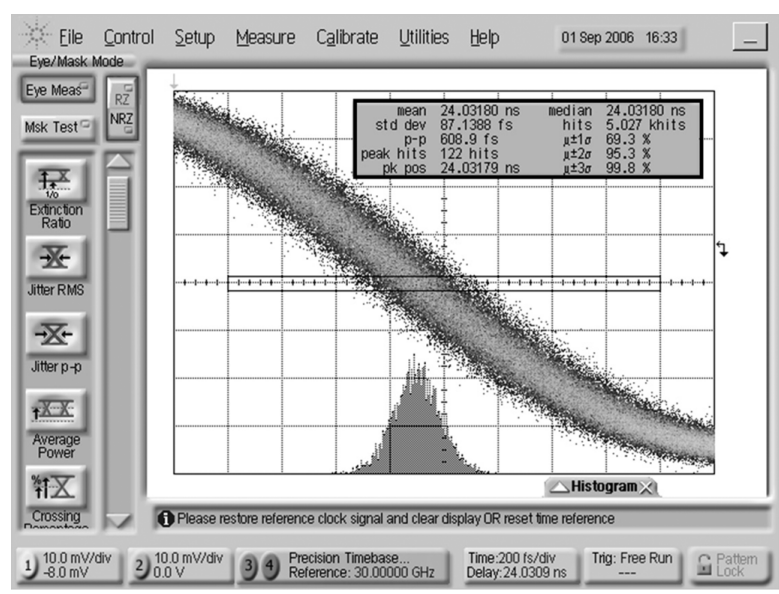

(a)

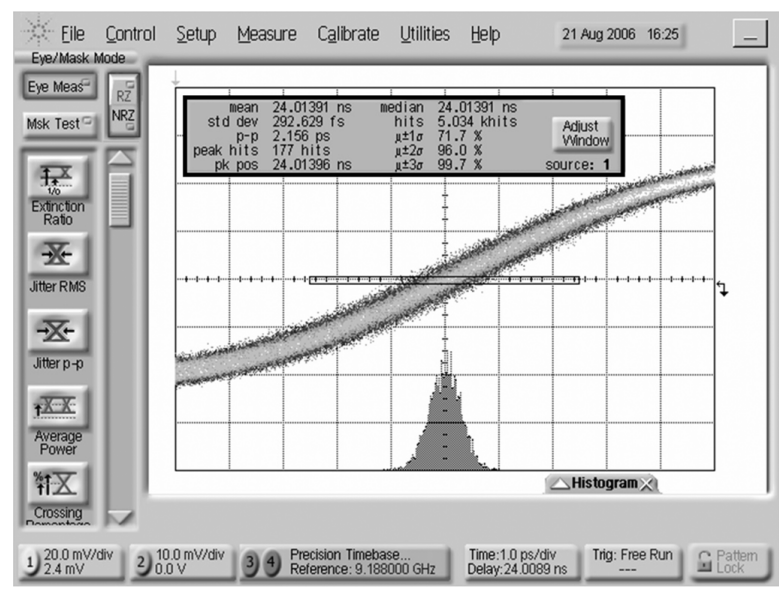

(b)

Fig. 22. Waveforms for (a) $75-\mathrm{GHz}$ and (b) $37.5-\mathrm{GHz}$ outputs.

\section{CONCLUSION}

A CMOS PLL with advanced millimeter-wave techniques demonstrates an operation frequency close to the device $f_{T}$. Novel VCO topology reveals significant improvement in terms of speed and noise, and the appropriate arrangement for dividers ensures locking along the loop. SSB-mixer-based PFD achieves truly quiet phase comparison, and therefore ultra low spurs. It not only provides technical solutions for present systems, but

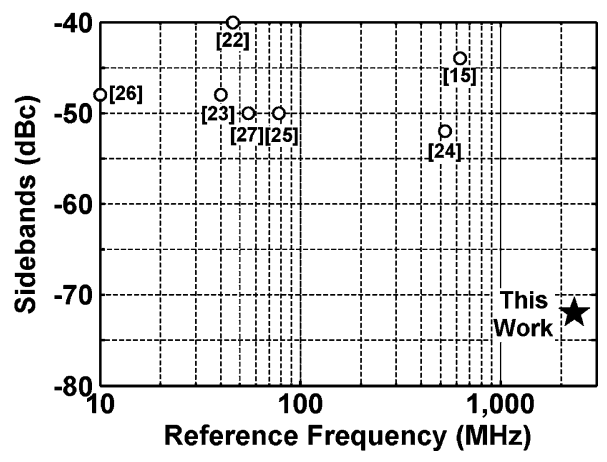

Fig. 23. Performance comparison of reference sidebands.

TABLE I

PLL PERFORMANCE SUMMARY

\begin{tabular}{|l|l|l|l|}
\hline & \multicolumn{1}{|c|}{ [27] } & \multicolumn{1}{c|}{ [28] } & \multicolumn{1}{c|}{ This Work } \\
\hline Operation Freq. & $60 \mathrm{GHz}$ & $60 \mathrm{GHz}$ & $75 \mathrm{GHz}$ \\
Multiply Ratio & 1024 & 12 & 32 \\
$\begin{array}{l}\text { Phase Noise } \\
\text { (@ 100-kHz Offset) }\end{array}$ & $-56 \mathrm{dBc} / \mathrm{Hz}$ & $-95.5 \mathrm{dBc} / \mathrm{Hz}$ & $-88 \mathrm{dBc} / \mathrm{Hz}$ \\
Clock Jitter & $\mathrm{N} / \mathrm{A}$ & $\mathrm{N} / \mathrm{A}$ & \\
@ 75 GHz & & & $87 \mathrm{fs}, \mathrm{rms} / 609 \mathrm{fs}, \mathrm{pp}$ \\
@ 37.5 GHz & & & $293 \mathrm{fs}, \mathrm{rms} / 2.15 \mathrm{ps}, \mathrm{pp}$ \\
Supply Voltage & $3 \mathrm{~V} / 4.3 \mathrm{~V}$ & $5 \mathrm{~V}$ & $1.45 \mathrm{~V}$ \\
Power Diss. & $650 \mathrm{~mW}$ & $370 \mathrm{~mW}$ & $88 \mathrm{~mW}$ \\
Ref. Sidebands & $-50 \mathrm{dBc}$ & $\mathrm{N} / \mathrm{A}$ & $<-72 \mathrm{dBc}$ \\
Min. Input Level & $-10 \mathrm{dBm}$ & $3 \mathrm{dBm}$ & $-16 \mathrm{dBm}$ \\
Lock Range With & $3.3 \mathrm{GHz}$ & $150 \mathrm{MHz}$ & $320 \mathrm{MHz}$ \\
Min. Input Level & & & \\
Loop Bandwidth & $200 \mathrm{kHz}$ & $178 \mathrm{MHz}$ & $2.5 \mathrm{MHz}$ \\
Chip Area & $1 \mathrm{~mm} \times 0.8 \mathrm{~mm}$ & $2.35 \mathrm{~mm} \times 1.8 \mathrm{~mm}$ & $1.0 \mathrm{~mm} \times 0.8 \mathrm{~mm}$ \\
Technology & $200-\mathrm{GHz}$ SiGe & $0.15-\mu \mathrm{m} \mathrm{GaAs}$ & $90-\mathrm{nm} \mathrm{CMOS}$ \\
\hline
\end{tabular}

* Reference and multiply ratio related.

also exhibits promising potential for future CMOS circuits operating beyond $100 \mathrm{GHz}$.

\section{REFERENCES}

[1] B. Razavi, "A 60-GHz CMOS receiver front-end," IEEE J. Solid-State Circuits, vol. 41, no. 1, pp. 17-22, Jan. 2006.

[2] C. H. Doan, S. Emami, A. M. Niknejad, and R. W. Brodersen, "Design of CMOS for $60 \mathrm{GHz}$ applications," in IEEE ISSCC 2004 Dig. Tech. Papers, Feb. 2004, pp. 440-441, 538.

[3] J. Lee, "High-speed circuit designs for transmitters in broadband data links," IEEE J. Solid-State Circuits, vol. 41, no. 5, pp. 1004-1015, May 2006.

[4] O. E. Akcasu, "High capacitance structure in a semiconductor device," U.S. Patent 5,208,725, May 4, 1993.

[5] K. Yamamoto and M. Fujishima, " $70 \mathrm{GHz}$ CMOS harmonic injectionlocked divider," in IEEE ISSCC 2006 Dig. Tech. Papers, Feb. 2006, pp. 600-601.

[6] G. Büren, C. Kromer, F. Ellinger, A. Huber, M. Schmatz, and H. Jackel, "A combined dynamic and static frequency divider for a 40GHz PLL in 80nm CMOS," in IEEE ISSCC 2006 Dig. Tech. Papers, Feb. 2006, pp. $598-599$.

[7] J. Lee and B. Razavi, "A 40-GHz frequency divider in 0.18- $\mu \mathrm{m} \mathrm{CMOS}$ technology," IEEE J. Solid-State Circuits, vol. 39, no. 4, pp. 594-601, Apr. 2004.

[8] J. Lee and B. Razavi, "A 40-Gb/s clock and data recovery circuit in 0.18- $\mu$ m CMOS technology," IEEE J. Solid-State Circuits, vol. 38, no. 12, pp. 2181-2190, Dec. 2003.

[9] A. Niknejad, ASITIC. UC Berkeley [Online]. Available: http://www. rfic.eecs.berkeley.edu/ niknejad/asitic.html

[10] Sonnet. Sonnet Software, Inc., Syracuse, NY [Online]. Available: http://www.sonnetusa.com 
[11] M. Mansuri and C. K. K. Yang, "A low-power adaptive bandwidth PLL and clock buffer with supply-noise compensation," IEEE J. Solid-State Circuits, vol. 38, no. 11, pp. 1804-1812, Nov. 2003.

[12] E. Hegazi, H. Sjoland, and A. A. Abidi, "A filtering technique to lower LC oscillator phase noise," IEEE J. Solid-State Circuits, vol. 36, no. 12, pp. 1921-1930, Dec. 2001.

[13] H. Wu and A. Hajimiri, "A $19 \mathrm{GHz} 0.5 \mathrm{~mW} 0.35 \mu \mathrm{m}$ CMOS frequency divider with shunt-peaking locking-range enhancement," in IEEE ISSCC 2001 Dig. Tech. Papers, Feb. 2001, pp. 412-413.

[14] A. Maxim et al., "A low jitter 125-1250 MHz process independent and ripple-poleless $0.18-\mu \mathrm{m}$ CMOS PLL based on a sample-reset loop filter," IEEE J. Solid-State Circuits, vol. 36, no. 11, pp. 1673-1683, Nov. 2001.

[15] J. Kim, J.-K. Kim, B. Lee, N. Kim, D. Jeong, and W. Kim, “A 20-GHz phase-locked loop for $40-\mathrm{Gb} / \mathrm{s}$ serializing transmitter in $0.13-\mu \mathrm{m}$ CMOS," IEEE J. Solid-State Circuits, vol. 41, no. 4, pp. 899-908, Apr. 2006.

[16] R. C. H. van de Beek et al., "A 2.5-10-GHz clock multiplier unit with 0.22-ps RMS jitter in standard 0.18- $\mu \mathrm{m}$ CMOS," IEEE J. Solid-State Circuits, vol. 39, no. 11, pp. 1862-1872, Nov. 2004.

[17] R. Gu et al., "A $6.25 \mathrm{GHz} 1 \mathrm{~V}$ LC-PLL in $0.13 \mu \mathrm{m} \mathrm{CMOS,"} \mathrm{in} \mathrm{IEEE}$ ISSCC 2006 Dig. Tech. Papers, Feb. 2006, pp. 594-595.

[18] A. Ng et al., "A 1V 24GHz 17.5mw PLL in 0.18/spl mu/m CMOS," in IEEE ISSCC 2005 Dig. Tech. Papers, Feb. 2005, vol. 1, pp. 158-590.

[19] D. Park and S. Mori, "Fast acquisition frequency synthesizer with the multiple phase detectors," in 1991 IEEE Pacific Rim Conf. Communications, Comput. Signal Process. Conf. Proc., Victoria, BC, Canada, May 1991, vol. 2, pp. 665-668.

[20] T. Lee and W. Lee, "A spur suppression technique for phase-locked frequency synthesizers," in IEEE ISSCC 2006 Dig. Tech. Papers, Feb. 2006, pp. 592-593.

[21] Maxim IC, "Clock (CLK) jitter and phase noise conversion," App. Note 3359, Dec. 10, 2004 [Online]. Available: http://www.maxim-ic.com/ appnotes.cfm/an_pk/3359

[22] C. Cao et al., "A 50-GHz phase-locked loop in 0.13- $\mu$ m CMOS," IEEE J. Solid-State Circuits, vol. 42, no. 8, pp. 1649-1656, Aug. 2007.

[23] T. Lin et al., "An agile VCO frequency calibration technique for a 10-GHz CMOS PLL," IEEE J. Solid-State Circuits, vol. 42, no. 2, pp. 340-349, Feb. 2007.

[24] G. Tak et al., "A 6.3-9-GHz CMOS fast settling PLL for MB-OFDM UWB applications," IEEE J. Solid-State Circuits, vol. 40, no. 8, pp. 1671-1679, Aug. 2005.

[25] Y. Ding et al., "A 21-GHz 8-modulus prescaler and a 20-GHz phaselocked loop fabricated in 130-nm CMOS," IEEE J. Solid-State Circuits, vol. 42, no. 6, pp. 1240-1249, Jun. 2007.

[26] N. Pavlovic, J. Gosselin, K. Mistry, and D. Leenaerts, "A $10 \mathrm{GHz}$ frequency synthesizer for 802.11a in 0.18- $\mu \mathrm{m}$ CMOS," in Proc. 30th Eur. Solid-State Circuits Conf. (ESSCIRC 2004), Sep. 2004, pp. 367-370.

[27] W. Winkler, J. Borngraber, B. Heinemann, and F. Herzel, "A fully integrated BiCMOS PLL for $60 \mathrm{GHz}$ wireless applications," in IEEE ISSCC 2005 Dig. Tech. Papers, Feb. 2005, pp. 406-407.
[28] J. Jeong and Y. Kwon, "A fully integrated V-band PLL MMIC using 0.15- $\mu \mathrm{m}$ GaAs phemt technology," IEEE J. Solid-State Circuits, vol. 41, no. 5, pp. 1042-1050, May 2006.

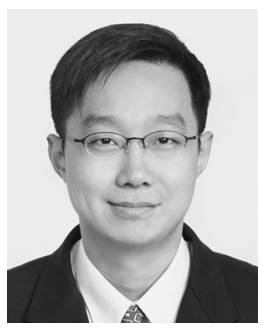

Jri Lee (S'03-M'04) received the B.Sc. degree in electrical engineering from National Taiwan University (NTU), Taipei, Taiwan in 1995, and the M.S. and Ph.D. degrees in electrical engineering from the University of California, Los Angeles (UCLA), both in 2003. His current research interests include high-speed wireless and wireline transceivers, phase-locked loops, and data converters.

After 2 years of military service (1995-1997), he was with Academia Sinica, Taipei, Taiwan from 1997 to 1998, and subsequently Intel Corporation from 2000 to 2002. He joined National Taiwan University (NTU) since 2004, where he is currently Associate Professor of electrical engineering. He is currently serving in the Technical Program Committees of the International Solid-State Circuits Conference (ISSCC), Symposium on VLSI Circuits, and Asian Solid-State Circuits Conference (A-SSCC). Prof. Lee received the Beatrice Winner Award for Editorial Excellence at the 2007 ISSCC, the Takuo Sugano Award for Outstanding Far-East Paper at the 2008 ISSCC, and NTU Outstanding Teaching Award in 2007.

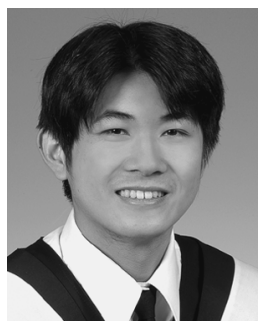

Mingchung Liu was born in Taipei, Taiwan, in 1982. $\mathrm{He}$ received the B.S. and M.S. degrees in electrical engineering from National Taiwan University, Taipei, Taiwan, in 2005 and 2007, respectively.

His research interests include broadband data communication circuits, phase-locked loops and clock and data recovery circuits.

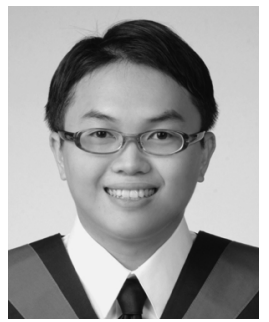

Huaide Wang was born in Taipei, Taiwan, in 1984. He received the B.S. degree in electrical engineering from National Taiwan University, Taipei, Taiwan, in 2006.

$\mathrm{He}$ is currently pursuing the Ph.D. degree in electrical engineering from the Graduate Institute of Electrical Engineering, National Taiwan University. His research interests are PLL and high-speed transceiver for wireline communication. 